Dobell, A. R., and Y. C. Ho

"An Optimal Unemployment Rate."

The Quarterly J ournal of Economics 81.4 (1967): pp. 675-683.

Reprinted with permission from

M.I.T. Press 


\title{
AN OPTIMAL UNEMPLOYMENT RATE *
}

\author{
A. R. Dobell and Y. C. Ho
}

I. Training costs and "unemployables," 675. - II. Solution, 678. - III. Assessment, 679. - Appendix, 680.

\section{Training Costs and "Unemployables"}

The concept of "overfull" employment has several connotations. One emerges from the literature on the Phillips-curve, concerned with the trade-off between lower unemployment and higher rates of increase of wages and prices. Another is the general notion of economic inflexibility and rigidity associated with unemployment rates below the level of "frictional" unemployment supposed to be necessary to lubricate the motion of a growing and changing economy. We leave these two considerations aside, in order to study a different issue, that of "unemployable" labor, of employment rates so high that remaining unemployed labor cannot profitably be brought into production.

The key consideration underlying this question is the cost of training. Qualifying people to enter employment costs resources, and probably more resources the higher is the present employment rate. This capital cost of training is captured in the model below, at the expense of some simplification in other directions. Then a question of obvious interest is whether the anticipated benefits from higher employment warrant the cost entailed. Since, however, the cost is in the nature of a capital cost, and the benefits more like flows, care has to be taken to ensure that the asset evaluations are correctly made. To carry out this exercise is the purpose of this note.

As our measure of the social benefit attached to any policy, we take the net consumption stream available to all individuals, employed or not, in the society. (Thus the distributional issues are set aside here; distribution is divorced from production. We want to know when it would profit the society to refuse to train labor for production, regardless of commitments on distribution of the re-

* Research of the first-named author was supported by the Harvard University Program on Technology and Society under a long-term grant from the IBM Corporation; research of the second-named author was supported in part under Joint Service Electronics Contract NONR 1866 (16) supported by the U.S. Army, Navy, and Air Force. While retaining sole responsibility for all conclusions, the authors wish to acknowledge this support with thanks. 
sulting consumption flow). Moreover, it is not just consumption now but consumption over some horizon which is of interest. We therefore pose the question as that of finding the social policy which yields a maximum to a criterion

$$
J=\int_{0}^{\infty}(C / L) \exp (-\gamma t) d t
$$

which represents the sum of all future per capita ${ }^{1}$ consumption flows, discounted for futurity at a positive social rate of discount $\gamma \cdot{ }^{2}$

The tools by which this social policy is shaped are assumed to be sharp enough to provide complete control over the proportion of resources flowing at any moment to consumption, investment in machines, and investment in training of labor. We suppose that these three flows exhaust gross national product, measured in physical terms (at supply prices), and also that it is legitimate to represent gross national product in physical terms as determined by a single production function having as inputs machines and trained, employed labor. Thus

$$
C+M+E=Q=F(K, W)
$$

where $C, M, E$, represent flows of output units to consumption, investment in machines, and education, respectively. ${ }^{3} F$ is a constant returns production function of the usual well-behaved sort, $K$ is the stock of machines, and $W$ is the supply of trained labor. It is assumed that untrained labor reverts to a subsistence or unemployment sector where it makes no contribution to output. (Such untrained, unemployed labor may still, of course, be fed at the average rate; as mentioned above, equal distribution of all output is consistent with the welfare criterion $J$.)

It is important to take account of the fact that machines and labor may be subject to differing rates of mortality and depreciation. Moreover, the costs of producing a machine and producing a trained laborer are different. Choosing units so that one unit of output transformed into capital goods yields one "machine," we then sup-

1. The variable $L$ actually represents the labor force, rather than total population. We follow custom in ignoring in this discussion the possibility of variable labor force participation rates, so the two values differ only by a constant multiple.

2. The implied linear utility function is overly simple, but the consequence of introducing a more general utility function is really only to reduce the rate at which the society travels along the solution paths derived below.

3 . This specialization to the case of a "one-sector" model is extreme, but sanctified by a long tradition in this growth literature. We use it here because it seems the only way to obtain specific conclusions without much more tedious discussion. 
pose that there is some function $d(w)$ which, for any employment rate $w(=W / L)$, gives the average capital cost per man to train additions to the work force. ${ }^{4}$ The problem, of course, is that this average cost is expected to rise as the employment rate rises toward 1.

Letting $n$ represent the rate of labor force (and population) growth, $\mu$ the rate of mortality or depreciation within the work force, and $\delta$ the rate of depreciation of machines, one can then summarize the growth of the model economy by the following equations showing the gross investment flows broken down into components representing net new additions and replacement.

$$
\begin{aligned}
& M=K^{\prime}+\delta K \\
& E=\left(W^{\prime}+\mu W\right) d \\
& C=F(K, W)-M-E,
\end{aligned}
$$

where primes denote time derivatives, and the argument $t$ is to be understood.

Social investment policy is to determine the gross investment expenditures $M$ and $E$ at each moment; as suggested earlier, this is to be done so as to yield a maximum to the social welfare criterion $J$. It is shown in the Appendix that these assumptions, together with a few obvious constraints, lead after some rewriting to the following problem, in which the capital/labor ratio, $r(=K / L)$, and the employment rate, $w(=W / L)$, appear as the key variables describing the state of the system, while $s(=M / Q)$ and $e(=E / Q)$ represent the policy instruments.

Maximize (by choosing investment rates $s(t), e(t),[0 \leqslant t]$ ) the value attained by the welfare criterion

$$
J=\int_{0}^{\infty}(1-s-e) w f(r / w) \exp (-\gamma t) d t
$$

subject to the constraints

$$
\begin{array}{ll}
r^{\prime}=s w f-(n+\delta) r & r(0)=r_{0} \\
w^{\prime}=e w f / d-(n+\mu) w & w(0)=w_{0}
\end{array}
$$

(where again primes denote derivatives with respect to time) and to

$$
\begin{aligned}
& 0 \leq s \leq 1 \\
& 0 \leq e \leq 1 \\
& 0 \leq s+e \leq 1 \\
& 0 \leq w \leq 1 \\
& 0 \leq r .
\end{aligned}
$$

4. Gestation lags in training are ignored. 


\section{Solution}

A full solution to this problem requires one to deal with a host of intricate transversality conditions, state-space constraints, and other issues familiar to the control theorist. Ho and Dobell tell the full story (for the case of constant training cost) elsewhere. ${ }^{5}$ But fortunately the results make sense to the economist even when the analysis itself may be of less interest. And the results can easily be summarized, even in the case of rising training costs.

In the graph below we plot $w$, the employment rate, against $r$, the capital/labor ratio.

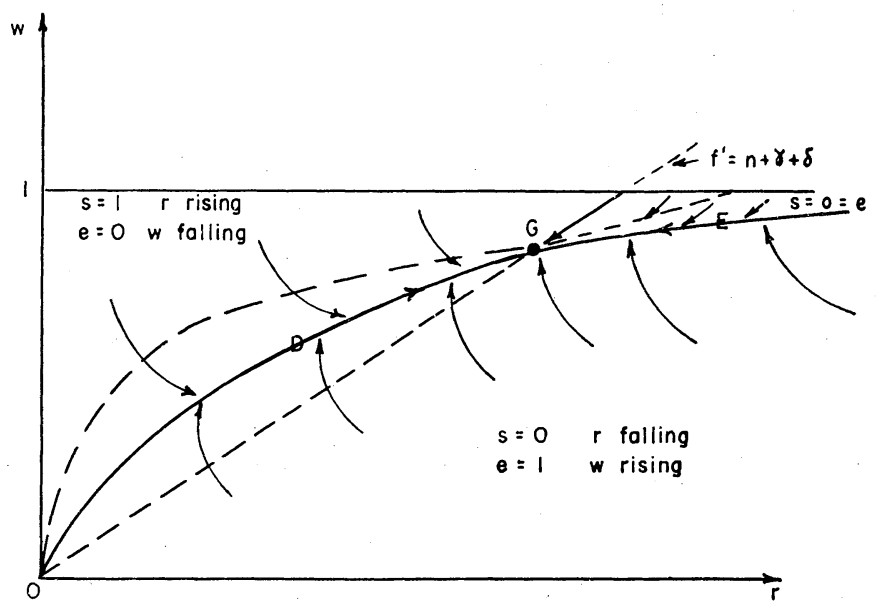

Figure I

The arrows tell the story. ${ }^{6}$ When the economy is above the path $O-D-G-E$, with lots of trained labor and relatively few machines, social policy dictates resources flowing toward increase of machines; below $O-D-G-E$, with relatively little trained labor, further training is indicated. (The reader may feel that, without an equation, he might have guessed so much; the remaining trick, though, is to determine exactly where the boundary falls.) Once the path $O-D-G-E$ is attained, expansion or decay along the path is indicated until finally the equilibrium point $G$ is reached. What, then, characterizes this path?

5. A. R. Dobell and Y. C. Ho, "Optimal Investment Policy: A Control Problem in Economic Theory," Institute of Electrical and Electronic Engineers, Transactions on Automatic Control AC 12 (Feb. 1967), pp. 4-14.

6. We have plotted a case in which the elasticity of the training cost curve is a constant. Other cases will differ in detail but not in principle. 
We note that along any ray through the origin the capital per worker is constant. Along the particular ray on which $G$ is located, the level of capital per worker is such that

$$
\partial F / \partial K-\delta=n+\gamma
$$

so that the net rate of return on machines (ignoring capital gains) just equals the effective social rate of discount on future (total) consumption. (This is therefore the "modified golden rule" capital/ worker ratio.) Above this ray the net rate of return on machines is higher, and accumulation of machines is therefore preferable to adding to present consumption. Hence the test of whether increasing employment is desirable is whether it is preferable to investing in machines; the path $O-D-G$ defines the point of balance on which net rates of return are equal, or in other words, on which

$$
(\partial F / \partial W) / d-\sigma(w)(n+\mu)-\mu=\partial F / \partial K-\delta,
$$

where $\sigma$ denotes the elasticity, $w d^{\prime}(w) / d(w)$, of the training cost function. Below the ray on which $G$ is located, the test of whether increasing employment is desirable is whether it is preferable to increasing consumption; the path $G-E$ is defined by

$$
(\partial F / \partial W) / d-\sigma(w)(n+\mu)-\mu=n+\gamma .
$$

The Appendix shows that these paths have the general shape indicated.

\section{Assessment}

Taking as the "rate of interest," $\rho$, in the economy the net rate of return on machines, if these are being produced, or the social rate of time preference, if consumption is being undertaken, then the above conditions are clearly plausible when the training cost $d$ is a constant; in that case they say simply that employment should be increased so long as wages (net of provision for mortality) in principle cover the implicit interest payments on the capital cost of training. When the training cost rises with employment, the above rule requires the net rate of return on trained labor to be further reduced by a term depending on the elasticity of the training cost function and on the gross rate of new entries to the labor force. (Such a correction would clearly be necessary in order to reflect rising marginal training costs.) Rephrasing the criterion, one could say that the rate of discount to be applied in capitalizing wages net of provision for replacement should be

$$
\rho+\sigma(w)(n+\mu)
$$

and that the society should permit employment to increase only so 
long as the capital value of a trained laborer so calculated does not fall short of the capital cost of training.

One intriguing consequence of this may be that a free market providing education loans at the going rate of interest in the economy cannot adequately reflect the externality of rising training costs. The profit-maximizing laborer, paying only the average cost of training, will surely borrow against future earnings for training now, so long as

$$
\partial F / \partial W-\mu d>\rho d
$$

But we know that he should be prevented from doing so, as soon as employment has risen to the point where

$$
\begin{aligned}
& \partial F / \partial W-\mu d=\rho d+d \sigma(n+\mu) \\
& =[\rho+\sigma(n+\mu)] d .
\end{aligned}
$$

Hence when training costs rise with $w$, the free capital market mechanism of loans for training could, in principle, lead to overinvestment in training, and an unemployment rate too low to be optimal.

Thus, in an extension of standard optimal accumulation models, we do find a criterion which tells us when increasing the employment rate would lead to lower consumption per capita and, in this model, a lower rate of growth. Beyond the boundary line described above, therefore, the social objectives of maximum employment are inevitably in conflict with those of maximum consumption or maximum growth. We have, as well, found a simplified example in which - in terms of the assumed criterion of social welfare - the case for a guaranteed annual income would be logically impeccable if the economy could thereby avoid inefficiently low unemployment rates.

\section{APPEndix}

The problem as phrased in the text was to select the levels of expenditures $C, M, E$ on consumption, investment in machines, and investment in training, respectively, in such a way that the social welfare criterion (1) attains its maximum subject to the specification $(2)$ to $(5)$.

Further constraints not mentioned in the text require that none of the gross flows $M, E, C$ (and neither $K$ nor $W$ ) may be negative, and require also that the employment rate $w=W / L$ not exceed 1 . Taking explicit account of these constraints, this problem could be approached directly by standard methods of optimal control. But it is more informative to reduce consideration instead to employment rates and capital/labor ratios. Therefore, expressing all investment flows as proportions of output and transforming to per capita terms, one can define 
and observe

$$
\begin{aligned}
& r=K / L \\
& w=W / L \\
& f(x)=F(x, 1)
\end{aligned}
$$

$$
\begin{array}{lc}
s=M / F(K, W) & 0 \leq s \leq 1 \\
e=E / F(K, W) & 0 \leq e \leq 1 \\
C / L=(1-s-e) F(K / L, W / L) & \\
=(1-s-e) w F(r / w, 1)=(1-s-e) w f(r / w) .
\end{array}
$$

Then recalling that

$$
\begin{aligned}
& r^{\prime}=K^{\prime} / L-n r=\operatorname{swf}(r / w)-(n+\delta) r \\
& w^{\prime}=W^{\prime} / L-n w=e w f(r / w) / d-(n+\mu) w
\end{aligned}
$$

and imposing the constraints mentioned above one has the problem described in equations (1) to (8).

A solution to this problem is given by Dobell and $\mathrm{Ho}^{7}$ for the case of constant training cost. Here the extension to the case of rising training cost will be briefly sketched.

We introduce implicit prices, $\exp (-\gamma t), \lambda_{r}, \lambda_{w}$, for the consumption good, the capital good, and trained labor respectively (all evaluated as at time zero, for delivery at time $t$ ) and form an expression differing only slightly from the implicit value per capita of net national product:

$$
\begin{aligned}
H & =(1-s-e) w f \exp (-\gamma t)+\lambda_{r}[s w f-(n+\delta) \mathrm{r}] \\
& +\left(\lambda_{w}+\eta\right)[\operatorname{ewf} / d-(n+\mu) w] \\
& =\left[(1-s-e) \exp (-\gamma t)+s \lambda_{r}+e\left(\lambda_{w}+\eta\right) / d\right] w f \\
& -\lambda_{r}(n+\delta) r-\left(\lambda_{w}+\eta\right)(n+\mu) w .
\end{aligned}
$$

From the theory of optimal accumulation we know it is necessary that the implicit asset prices satisfy the usual arbitrage conditions: ${ }^{8}$

$$
\begin{aligned}
\lambda_{r}^{\prime} & =-a f^{\prime}(r / w)+(n+\delta) \lambda_{r}=-\partial H / \partial r \\
\lambda^{\prime}{ }_{w} & =-a Z+(n+\mu)\left(\lambda_{w}+\eta\right) \\
& +\left(\lambda_{w}+\eta\right) \text { ef } \sigma(w) / d=-\partial H / \partial w
\end{aligned}
$$

and analogy with the finite horizon case suggests in addition the terminal conditions

where

$$
\begin{aligned}
& \lambda_{r} \rightarrow 0 \text { as } t \rightarrow \infty \\
& \left(\lambda_{w}+\eta\right) \rightarrow 0 \text { as } t \rightarrow \infty,
\end{aligned}
$$

$$
\begin{aligned}
& \eta \equiv 0 \text { if } w<1 \\
& \eta \leq 0 \text { if } w=1
\end{aligned}
$$$$
a=\left[(1-s-e) \exp (-\gamma t)+s \lambda_{r}+e\left(\lambda_{w}+\eta\right) / d\right]
$$$$
\boldsymbol{\sigma}(w)=w d^{\prime}(w) / d(w) \text {. }
$$$$
Z=f-f^{\prime}(r / w) r / w=\partial F / \partial W \text {. }
$$

The full employment cases where $w \equiv 1$ do not differ significantly from the usual one-dimensional optimal accumulation problem ${ }^{9}$

7. Op. cit.

8. For notational simplicity, primes will denote differentiation with respect to the argument shown. Where no argument appears, primes will continue to denote differentiation with respect to time. The argument $r / w$ will frequently be omitted from the function $f$, and the argument $w$ from the function $d$.

9. See D. Cass, "Optimum Growth in an Aggregative Model of Capital Accumulation," Review of Economic Studies, XXXII (July 1965), 233-40. 
and have been fully discussed by the authors. ${ }^{1}$ We may therefore confine attention to the case of less than full employment, where $\eta \equiv 0$. In particular, it is reasonable to consider only the (singular) cases in which the benefits promised by the investment alternatives are in balance with each other or with the benefits obtainable from consumption. Such cases are signaled by equality of two or more benefit/cost ratios for the options open.

The path $O-D-G$, for example, is defined by the condition

$$
\text { D: } \quad \lambda_{w} / d \equiv \lambda_{r}
$$

which requires

$$
\lambda_{w}^{\prime}-\lambda_{w} \sigma(w) w^{\prime} / w \equiv \lambda_{r}^{\prime} d
$$

and, substituting from above, one obtains

$$
Z / d-\mu-\sigma(w)(n+\mu) \equiv f^{\prime}(r / w)-\delta .
$$

The path $G-E$ is defined by

$$
\begin{array}{ll}
E: & \lambda_{w} / d \equiv \exp (-\gamma t) \\
\text { or } & \lambda^{\prime}{ }_{w}-\lambda_{w} \sigma(w) w^{\prime} / w \equiv-\gamma \lambda_{w}
\end{array}
$$

which yields

$$
Z / d-\mu-\sigma(w)(n+\mu) \equiv \gamma+n .
$$

The equilibrium state $G$ is defined by

$$
G: \quad \lambda_{w} / d \equiv \lambda_{r} \equiv \exp (-\gamma t)
$$

which requires

$$
f^{\prime}-\delta=Z / d-\mu-\sigma(w)(n+\mu)=n+\gamma .
$$

It can easily be checked - using only the assumptions of diminishing marginal products and rising training costs - that the locus of points defined by $D$ intersects that defined by $E$ at the point $G$; that above the ray on which $G$ lies, where $f^{\prime}-\delta>n+\gamma$, the path $E$ lies above $D$; that where $f^{\prime}-\delta<n+\gamma$, the path $E$ lies below $D$. (This applies, of course, to the case where some unemployment exists; once either path attains $w=1$, the full employment analysis applies, and the paths $D$ and $E$ may coincide with the line $w=1$ ). Moreover, if $\sigma(w)$ is taken to be a constant - and in fact so long as $\sigma^{\prime}(w)$ is not too strongly negative - it can be shown that the curves defined by $D$ and $E$ are rising. When $d^{\prime}(w)$ is zero, they are both rays through the origin, with $D$ closer to the ray on which $G$ lies. For an example in which $F$ is a Cobb-Douglas function, $\sigma$ constant, the two curves are also concave, but we have not found any meaningful economic restrictions which would guarantee this property in general. We have plotted the curves appropriate to this example in constructing Figure I.

It may, finally, be checked that from any initial point a trajectory following the arrows on Figure I can be generated by suitable choice of initial prices, with $s(t), e(t)$ determined at each moment so as to maximize the implicit value of net national product per capita, and will satisfy all the above necessary conditions for an optimal trajectory, and the added terminal conditions as well. It can be shown that these conditions are sufficient to guarantee that no other feasible path can provide a higher value for the criterion $J$.

1. Op. cit. 
The reader interested in greater detail than we considered appropriate in this note may refer to the more technical discussion by the authors, ${ }^{2}$ and to the sufficiency proof given by them. ${ }^{3}$

2. Op. cit.

3. A. R. Dobell and Y. C. Ho, "A Control Problem in Economic Theory," in Mathematical Systems Theory and Economics, Proceedings of a Conference at Varenna, Italy, June 1967.

HaRvard UNIVERSity 